\title{
Regioselective and Diastereoselective Amination using Chlorosulfonyl Isocyanate: A Short and Efficient Synthesis of (-)-Cytoxazone
}

Ji Duck Kim, In Su Kim, Cheng Hua Jin, Ok Pyo Zee and Young Hoon Jung*

\section{General Methods}

Commercially available reagents were used without additional purification, unless otherwise stated. All anhydrous solvents were distilled over $\mathrm{CaH}_{2}$ or $\mathrm{P}_{2} \mathrm{O}_{5}$ or $\mathrm{Na}$ / benzophenone prior to reaction. All reactions were performed under an inert atmosphere of nitrogen or argon. Melting points were measured on a Gallenkamp melting point apparatus or Electrothermal IA9300 melting point apparatus and were not corrected. Nuclear magnetic resonance spectra $\left({ }^{1} \mathrm{H}\right.$ and $\left.{ }^{13} \mathrm{C} \mathrm{NMR}\right)$ were recorded on a Varian Unity Inova $500 \mathrm{MHz}$ spectrometer for $\mathrm{CDCl}_{3}$ solutions and chemical shifts are reported as parts per million (ppm) relative to, respectively, residual $\mathrm{CHCl}_{3} \delta_{\mathrm{H}}(7.26 \mathrm{ppm})$ and $\mathrm{CDCl}_{3} \delta_{\mathrm{C}}(77.0 \mathrm{ppm})$ as internal standards. Resonance patterns are reported with the notations s (singlet), d (doublet), t (triplet), q (quartet), and $\mathrm{m}$ (multiplet). In addition, the notation br is used to indicate a broad signal. Coupling constants $(J)$ are reported in hertz $(\mathrm{Hz})$. IR spectra were recorded on a Nicolet 205 Infrared spectrophotometer or Bruker Vector 22 Infrared spectrophotometer and are reported as $\mathrm{cm}^{-1}$. Optical rotations were measured with a Jasco P1020 polarimeter. Thin layer chromatography was carried out using plates coated with Kieselgel $60 \mathrm{~F}_{254}$ (Merck). For flash column chromatography, E. Merck Kieselgel 60 (230-400 mesh) was used. High-resolution mass spectra (HRMS) were recorded on a JEOL, JMS-505 or JMS600 spectrometer using the chemical ionization (CI) method.

\section{Synthesis of $p$-Methoxybenzylic Ethers}

\section{(1R,2S)-1,2-Dimethoxy-1-p-methoxyphenylbut-3-ene (3).}

(1R,2S)-1-p-Methoxyphenylbut-3-ene-1,2-diol (9)

To a solution of allyl(diisopropylamino)dimethylsilane ${ }^{10}(5.10 \mathrm{~mL}, 20.86 \mathrm{mmol})$ in anhydrous $\mathrm{Et}_{2} \mathrm{O}(25$ $\mathrm{mL})$ was added TMEDA $(3.15 \mathrm{~mL}, 20.86 \mathrm{mmol})$ and $n$-butyllithium (13.04 mL, 20.86 mmol, $1.6 \mathrm{M}$ in hexane) at $0{ }^{\circ} \mathrm{C}$ under $\mathrm{N}_{2}$. The solution was kept at $0^{\circ} \mathrm{C}$ for $4 \mathrm{~h}$ and cooled to $-78{ }^{\circ} \mathrm{C}$. The reaction mixture was treated with (-)-B-methoxydiisopinocampheylborane $(7.85 \mathrm{~g}, 24.83 \mathrm{mmol})$ in anhydrous $\mathrm{Et}_{2} \mathrm{O}(5 \mathrm{~mL})$ and stirred at $78^{\circ} \mathrm{C}$ for $2 \mathrm{~h}$. To this solution was added boron trifluoride etherate $(3.43 \mathrm{~mL}, 27.03 \mathrm{mmol})$ and a solution of $p$ anisaldehyde $(2.00 \mathrm{~g}, 14.69 \mathrm{mmol})$ in anhydrous $\mathrm{Et}_{2} \mathrm{O}(5 \mathrm{~mL})$. The reaction mixture was kept at $-78{ }^{\circ} \mathrm{C}$ for $3 \mathrm{~h}$. To this mixture was added THF (20 mL), MeOH (20 mL), KF (2.43 g, $41.87 \mathrm{mmol}), \mathrm{KHCO}_{3}(4.19 \mathrm{~g}, 41.87$ $\mathrm{mmol})$ and $30 \% \mathrm{H}_{2} \mathrm{O}_{2}(45 \mathrm{~mL})$. The reaction mixture was stirred at room temperature for $20 \mathrm{~h}$ and cooled to 0 
${ }^{\circ} \mathrm{C}$, and the excess $\mathrm{H}_{2} \mathrm{O}_{2}$ was quenched by the addition of $\mathrm{Na}_{2} \mathrm{~S}_{2} \mathrm{O}_{3}$. The mixture was diluted with EtOAc (100 $\mathrm{mL}$ ) and filtered through celite pad. The celite pad was wahed with EtOAc and the filtrate was dried over $\mathrm{MgSO}_{4}$ and concentrated in vacuo. The residue was purified by column chromatography $($ Hexane/EtOAc $=1: 1)$ and recrystallization (Toluene) to give $1.48 \mathrm{~g}(52 \%)$ of (1R,2S)-1-p-methoxyphenylbut-3-ene-1,2-diol (9) as a colorless crystal.

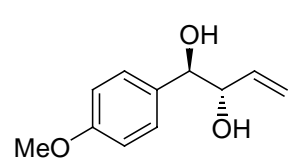

$\mathrm{R}_{\mathrm{f}} 0.25\left(1: 1\right.$ Hexane/EtOAc); mp 87 89 ${ }^{\circ} \mathrm{C} ;[\alpha]_{\mathrm{D}}^{25}-73.2^{\circ}\left(c 0.1, \mathrm{CDCl}_{3}\right)$; IR $\left(\mathrm{CH}_{2} \mathrm{Cl}_{2}\right)$ : 3408, 2954, 2837, 1612, 1514, $1463 \mathrm{~cm}^{-1} ;{ }^{1} \mathrm{H}$ NMR (500 MHz, $\mathrm{CDCl}_{3}$ ): $\delta$ 1.93-1.99 (br, 1H), 2.30-2.37 (br, 1H), 3.81 (s, 3H), 4.25-4.33 (br, 1H), 4.66-4.70 (br, 1H), 5.24 (dd, 1H, $J$ $=10.5,1.5 \mathrm{~Hz}), 5.31(\mathrm{dd}, 1 \mathrm{H}, J=17.0,1.5 \mathrm{~Hz}), 5.83(\mathrm{ddd}, 1 \mathrm{H}, J=17.0,10.5,6.5 \mathrm{~Hz}), 6.89(\mathrm{dd}, 2 \mathrm{H}, J=7.0$, $2.0 \mathrm{~Hz}), 7.29(\mathrm{dd}, 2 \mathrm{H}, J=7.0,2.0 \mathrm{~Hz}) ;{ }^{13} \mathrm{C} \mathrm{NMR}\left(125 \mathrm{MHz}, \mathrm{CDCl}_{3}\right): \delta 55.52,76.44,77.53,114.03,118.07$, 128.19, 132.12, 136.36, 159.59; Anal. Calcd for $\mathrm{C}_{11} \mathrm{H}_{14} \mathrm{O}_{3}: \mathrm{C}, 68.02$; H, 7.27. Found: C, 68.22; H, 7.29.

(1R,2S)-1,2-Dimethoxy-1-p-methoxyphenylbut-3-ene (3).

To a solution of (1R,2S)-1-p-methoxyphenylbut-3-ene-1,2-diol (9) (1.20 g, $6.18 \mathrm{mmol})$ in anhydrous THF $(25 \mathrm{~mL})$ was added $\mathrm{NaH}(0.54 \mathrm{~g}, 13.59 \mathrm{mmol}, 60 \%$ in mineral oil $)$ and $\mathrm{MeI}(1.15 \mathrm{~mL}, 18.53 \mathrm{mmol})$ at $0{ }^{\circ} \mathrm{C}$ under $\mathrm{N}_{2}$. The reaction mixture was stirred at $0{ }^{\circ} \mathrm{C}$ for $3 \mathrm{~h}, \mathrm{H}_{2} \mathrm{O}(30 \mathrm{~mL})$ was added and the solution was extracted with EtOAc $(50 \mathrm{~mL})$. The organic layer was washed with $\mathrm{H}_{2} \mathrm{O}$ and brine, dried over $\mathrm{MgSO}_{4}$ and concentrated in vacuo. The residue was purified by column chromatography (Hexane/EtOAc $=10: 1)$ to afford $1.32 \mathrm{~g}(96 \%)$ of $(1 R, 2 S)$-1,2-dimethoxy-1-p-methoxyphenylbut-3-ene (3) as a colorless oil.

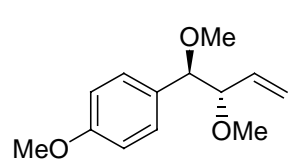

$\mathrm{R}_{\mathrm{f}} 0.40$ (6:1 Hexane/EtOAc); $[\alpha]_{\mathrm{D}}^{25}-49.3^{\circ}$ (c 0.2, $\left.\mathrm{CDCl}_{3}\right)$; IR (neat): 2934, 2822, 1612 , 1512, $1464 \mathrm{~cm}^{-1} ;{ }^{1} \mathrm{H}$ NMR (500 MHz, $\left.\mathrm{CDCl}_{3}\right): \delta 3.23(\mathrm{~s}, 3 \mathrm{H}), 3.24$ (s, 3H), $3.65(\mathrm{dd}, 1 \mathrm{H}, J$ $=8.0,5.0 \mathrm{~Hz}), 3.81(\mathrm{~s}, 3 \mathrm{H}), 4.80(\mathrm{~d}, 1 \mathrm{H}, J=5.0 \mathrm{~Hz}), 5.15(\mathrm{dd}, 1 \mathrm{H}, J=17.5,1.5 \mathrm{~Hz}), 5.27$

$(\mathrm{dd}, 1 \mathrm{H}, J=10.0,1.5 \mathrm{~Hz}), 5.74(\mathrm{ddd}, 1 \mathrm{H}, J=17.5,10.0,8.0 \mathrm{~Hz}), 6.88(\mathrm{dd}, 2 \mathrm{H}, J=6.5,2.0 \mathrm{~Hz}), 7.23(\mathrm{dd}, 2 \mathrm{H}$, $J=6.5,2.0 \mathrm{~Hz}) ;{ }^{13} \mathrm{C} \mathrm{NMR}\left(125 \mathrm{MHz}, \mathrm{CDCl}_{3}\right): \delta 55.19,56.80,56.95,85.51,86.33,113.41,119.17,128.99$, 130.58, 134.69, 159.14; Anal. Calcd for $\mathrm{C}_{13} \mathrm{H}_{18} \mathrm{O}_{3}$ : C, 70.24; H, 8.16. Found: C, 70.33; H, 8.04.

\section{(1R,2R)-1-Methoxy-1-p-methoxyphenyl-2-methylbut-3-ene (5).}

(1R,2R)-1-p-Methoxyphenyl-2-methylbut-3-en-1-ol

To a suspension of $t$-BuOK $(0.58 \mathrm{~g}, 5.14 \mathrm{mmol})$ in anhydrous THF $(5 \mathrm{~mL})$ was added excess of trans-2butene and $n$-butyllithium $\left(3.21 \mathrm{~mL}, 5.14 \mathrm{mmol}, 1.6 \mathrm{M}\right.$ in hexane) at $-78{ }^{\circ} \mathrm{C}$ under $\mathrm{N}_{2}$. The reaction mixture was 
stirred at $-45{ }^{\circ} \mathrm{C}$ for $10 \mathrm{~min}$ and recooled to $-78^{\circ} \mathrm{C}$. To this mixture was added (+)- $B$ methoxydiisopinocampheylborane $(1.95 \mathrm{~g}, 6.17 \mathrm{mmol})$ in anhydrous $\mathrm{Et}_{2} \mathrm{O}(3 \mathrm{~mL})$ at $-78{ }^{\circ} \mathrm{C}$. After the reaction mixture was stirred at $-78{ }^{\circ} \mathrm{C}$ for $30 \mathrm{~min}$, boron trifluoride etherate $(0.87 \mathrm{~mL}, 6.89 \mathrm{mmol})$ and a solution of $p$ anisaldehyde $(0.70 \mathrm{~g}, 5.14 \mathrm{mmol})$ in anhydrous $\mathrm{Et}_{2} \mathrm{O}(3 \mathrm{~mL})$. The reaction mixture was stirred $-78{ }^{\circ} \mathrm{C}$ for $3 \mathrm{~h}$, and then treated with $3 \mathrm{~N} \mathrm{NaOH}(3.76 \mathrm{~mL})$ and $30 \% \mathrm{H}_{2} \mathrm{O}_{2}(1.54 \mathrm{~mL})$, and the mixture was refluxed for $1 \mathrm{~h}$. The organic layer was separated, washed with $\mathrm{H}_{2} \mathrm{O}$ and brine, dried over $\mathrm{MgSO}_{4}$ and concentrated in vacuo. The residue was purified by column chromatography (Hexane/EtOAc $=6: 1)$ to give $0.64 \mathrm{~g}(65 \%)$ of $(1 R, 2 R)-1-p$ methoxyphenyl-2-methylbut-3-en-1-ol as a colorless oil.

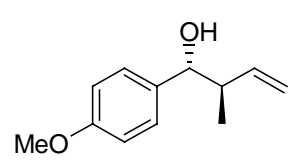

$\mathrm{R}_{\mathrm{f}} 0.40$ (3:1 Hexane/EtOAc); $[\alpha]_{\mathrm{D}}{ }^{25}+43.2^{\circ}\left(c 0.2, \mathrm{CDCl}_{3}\right)$; IR (neat): 3432, 2962, 2836, 1612, 1513, $1461 \mathrm{~cm}^{-1} ;{ }^{1} \mathrm{H}$ NMR (500 MHz, $\left.\mathrm{CDCl}_{3}\right): \delta 0.97$ (d, 3H, $\left.J=6.5 \mathrm{~Hz}\right), 2.06-2.11$ (br d, 1H, $J=2.5 \mathrm{~Hz}), 2.47$ (ddq, 1H, $J=8.0,8.0,6.5 \mathrm{~Hz}), 3.82(\mathrm{~s}, 3 \mathrm{H}), 4.33$ (dd, $1 \mathrm{H}, J=$ 8.0, $2.5 \mathrm{~Hz}), 5.19(\mathrm{dd}, 1 \mathrm{H}, J=11.0,1.5 \mathrm{~Hz}), 5.23(\mathrm{dd}, 1 \mathrm{H}, J=17.0,1.5 \mathrm{~Hz}), 5.84(\mathrm{ddd}, 1 \mathrm{H}, J=17.0,11.0,8.0$ $\mathrm{Hz}), 6.91(\mathrm{dd}, 2 \mathrm{H}, J=7.0,2.0 \mathrm{~Hz}), 7.26(\mathrm{dd}, 2 \mathrm{H}, J=7.0,2.0 \mathrm{~Hz}) ;{ }^{13} \mathrm{C}$ NMR $\left(125 \mathrm{MHz}, \mathrm{CDCl}_{3}\right): \delta 16.82$, 46.63, 55.49, 77.75, 113.91, 116.89, 128.22, 134.86, 141.19, 159.39; HRMS (CI) calcd for $\mathrm{C}_{12} \mathrm{H}_{16} \mathrm{O}_{2}+\mathrm{H}$ $(\mathrm{M}+\mathrm{H})^{+}$193.1228. Found: 193.1226.

(1R,2R)-1-Methoxy-1-p-methoxyphenyl-2-methylbut-3-ene (5).

The similar procedure for 3 was followed using (1R,2R)-1-p-methoxyphenyl-2-methylbut-3-en-1-ol $(0.50 \mathrm{~g}$, $2.60 \mathrm{mmol}), \mathrm{NaH}(0.11 \mathrm{~g}, 2.86 \mathrm{mmol}, 60 \%$ in mineral oil) and $\mathrm{MeI}(0.24 \mathrm{~mL}, 3.90 \mathrm{mmol})$ in THF $(10 \mathrm{~mL})$. The reaction mixture was purified by column chromatography (Hexane/EtOAc $=30: 1)$ to give $0.51 \mathrm{~g}(95 \%)$ of $(1 R, 2 R)-1$-methoxy-1-p-methoxyphenyl-2-methylbut-3-ene (5) as a colorless oil.

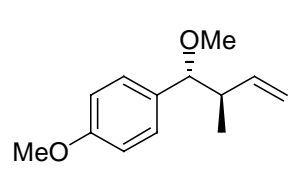

$\mathrm{R}_{\mathrm{f}} 0.40(15: 1 \mathrm{Hexane} / \mathrm{EtOAc}) ;[\alpha]_{\mathrm{D}}^{25}+82.8^{\circ}\left(c\right.$ 0.2, $\left.\mathrm{CDCl}_{3}\right)$; IR (neat): 2976, 2932, 2835, 1612, 1512, $1463 \mathrm{~cm}^{-1} ;{ }^{1} \mathrm{H}$ NMR (500 MHz, $\left.\mathrm{CDCl}_{3}\right): \delta 0.85$ (d, 3H, $J=7.0 \mathrm{~Hz}$ ), 2.52 (ddq, $1 \mathrm{H}, J=8.0,7.0,7.0 \mathrm{~Hz}), 3.19(\mathrm{~s}, 3 \mathrm{H}), 3.84(\mathrm{~s}, 3 \mathrm{H}), 3.88(\mathrm{~d}, 1 \mathrm{H}, J=8.0 \mathrm{~Hz}), 5.04(\mathrm{dd}, 1 \mathrm{H}, J$ $=10.5,1.5 \mathrm{~Hz}), 5.06(\mathrm{dd}, 1 \mathrm{H}, J=16.5,1.5 \mathrm{~Hz}), 5.91(\mathrm{ddd}, 1 \mathrm{H}, J=16.5,10.5,7.0 \mathrm{~Hz}), 6.90(\mathrm{dd}, 2 \mathrm{H}, J=6.5$, $2.0 \mathrm{~Hz}), 7.19(\mathrm{dd}, 2 \mathrm{H}, J=6.5,2.0 \mathrm{~Hz}) ;{ }^{13} \mathrm{C} \mathrm{NMR}\left(125 \mathrm{MHz}, \mathrm{CDCl}_{3}\right): \delta 16.63,44.58,55.45,56.96,87.99$, 113.73, 114.39, 128.85, 132.73, 141.71, 159.29; HRMS (CI) calcd for $\mathrm{C}_{13} \mathrm{H}_{18} \mathrm{O}_{2}+\mathrm{H}(\mathrm{M}+\mathrm{H})^{+}$207.1385. Found: 207.1387.

\section{Reaction of $p$-Methoxybenzylic Ethers with CSI}




\section{General procedure for the reaction of $p$-methoxybenzylic ether with CSI.}

A suspension of $\mathrm{Na}_{2} \mathrm{CO}_{3}(6.75 \mathrm{mmol})$ in anhydrous solvent $(12 \mathrm{~mL})$ was adjusted to $-78{ }^{\circ} \mathrm{C}$, then $\mathrm{CSI}(4.50$ mmol) and $p$-methoxybenzylic ether $(3.00 \mathrm{mmol})$ was added under $\mathrm{N}_{2}$. The reaction mixture was stirred at -78 ${ }^{\circ} \mathrm{C}$, quenched with $\mathrm{H}_{2} \mathrm{O}(10 \mathrm{~mL})$ when the reaction was completed (TLC monitoring), then extracted with EtOAc $(10 \mathrm{~mL} \times 2)$. The organic layer was added to an aqueous solution of $\mathrm{Na}_{2} \mathrm{SO}_{3}(25 \%)$ and $\mathrm{KOH}(10 \%)$, and the reaction mixture was stirred at room temperature for overnight. The organic layer was washed with $\mathrm{H}_{2} \mathrm{O}$ and brine, dried over $\mathrm{MgSO}_{4}$ and concentrated in vacuo. The residue was purified by column chromatography (Hexane/EtOAc).

\section{$(1 R, 2 S)$-Methyl $N$-(2-methoxy-1-p-methoxyphenylbut-3-enyl)carbamate (2).}

The above general procedure was followed using (1R,2S)-1,2-dimethoxy-1-p-methoxyphenylbut-3-ene $(3)$ $(0.70 \mathrm{~g}, 3.15 \mathrm{mmol}), \mathrm{Na}_{2} \mathrm{CO}_{3}(1.10 \mathrm{~g}, 10.39 \mathrm{mmol})$ and $\mathrm{CSI}(0.60 \mathrm{~mL}, 6.93 \mathrm{mmol})$ in toluene $(13 \mathrm{~mL})$ at $-78{ }^{\circ} \mathrm{C}$. The reaction mixture was purified by column chromatography (Hexane/EtOAc $=3: 1)$ to give $0.79 \mathrm{~g}(95 \%)$ of (1R,2S)-methyl $N$-(2-methoxy-1-p-methoxyphenylbut-3-enyl)carbamate (2) (27: 1 ratio) as a white solid.

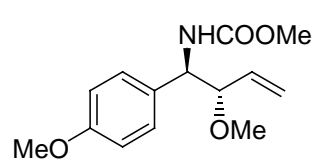

$\mathrm{R}_{\mathrm{f}} 0.28\left(3: 1\right.$ Hexane/EtOAc); mp 93 95 ${ }^{\circ} \mathrm{C} ;[\alpha]_{\mathrm{D}}{ }^{25}-22.4^{\circ}\left(c 0.2, \mathrm{CDCl}_{3}\right) ; \mathrm{IR}\left(\mathrm{CH}_{2} \mathrm{Cl}_{2}\right)$ : 3330, 2938, 2836, 1710, 1612, 1514, $1464 \mathrm{~cm}^{-1} ;{ }^{1} \mathrm{H}$ NMR $\left(500 \mathrm{MHz}, \mathrm{CDCl}_{3}\right): \delta 3.32$ (s, $3 \mathrm{H}), 3.66$ (s, 3H), 3.81 (s, 3H), 3.86-3.93 (br, 1H), 4.72-4.80 (br, 1H), 5.26 (dd, 1H, $J=$ 11.0, $2.0 \mathrm{~Hz}), 5.29(\mathrm{dd}, 1 \mathrm{H}, J=17.5,2.0 \mathrm{~Hz}), 5.35-5.42(\mathrm{~m}, 1 \mathrm{H}), 5.50-5.60(\mathrm{br}, 1 \mathrm{H}), 6.86(\mathrm{dd}, 2 \mathrm{H}, J=8.0,2.0$ $\mathrm{Hz}), 7.23(\mathrm{dd}, 2 \mathrm{H}, J=8.0,2.0 \mathrm{~Hz}) ;{ }^{13} \mathrm{C} \mathrm{NMR}\left(125 \mathrm{MHz}, \mathrm{CDCl}_{3}\right): \delta 52.37,55.45,57.08,57.72,85.08,113.77$, 119.71, 129.30, 131.17, 135.06, 156.52, 159.10; Anal. Calcd for $\mathrm{C}_{14} \mathrm{H}_{19} \mathrm{NO}_{4}$ : C, 63.38; H, 7.22; N, 5.28. Found: C, 63.50; H, 7.25; N, 5.33.

The above general procedure was followed using (1R,2S)-1,2-dimethoxy-1-p-methoxyphenylbut-3-ene (3) (50 mg, $0.23 \mathrm{mmol}), \mathrm{Na}_{2} \mathrm{CO}_{3}(79 \mathrm{mg}, 0.74 \mathrm{mmol})$ and $\mathrm{CSI}(43 \mu \mathrm{L}, 0.49 \mathrm{mmol})$ in $\mathrm{CH}_{2} \mathrm{Cl}_{2}(2 \mathrm{~mL})$ at $0{ }^{\circ} \mathrm{C}$. The reaction mixture was purified by column chromatography (Hexane/EtOAc $=3: 1$ ) to give $56 \mathrm{mg}(94 \%)$ inseparable mixture of $(1 R, 2 S)$-methyl $N$-(2-methoxy-1-p-methoxyphenylbut-3-enyl)carbamate (2) and $(1 S, 2 S)$ methyl $N$-(2-methoxy-1-p-methoxyphenylbut-3-enyl)carbamate (4) (5.7 : 1 ratio) as a white solid.

The above general procedure was followed using (1R,2S)-1,2-dimethoxy-1-p-methoxyphenylbut-3-ene (3) (50 mg, $0.23 \mathrm{mmol}), \mathrm{Na}_{2} \mathrm{CO}_{3}(79 \mathrm{mg}, 0.74 \mathrm{mmol})$ and CSI (43 $\left.\mu \mathrm{L}, 0.49 \mathrm{mmol}\right)$ in $\mathrm{CH}_{2} \mathrm{Cl}_{2}(2 \mathrm{~mL})$ at $-78{ }^{\circ} \mathrm{C}$. The 
reaction mixture was purified by column chromatography (Hexane/EtOAc $=3: 1)$ to give $55 \mathrm{mg}(92 \%)$ inseparable mixture of $(1 R, 2 S)$-methyl $N$-(2-methoxy-1-p-methoxyphenylbut-3-enyl)carbamate (2) and $(1 S, 2 S)$ methyl $N$-(2-methoxy-1-p-methoxyphenylbut-3-enyl)carbamate (4) (7.0:1 ratio) as a white solid.

The above general procedure was followed using $(1 R, 2 S)$-1,2-dimethoxy-1-p-methoxyphenylbut-3-ene (3) (50 mg, $0.23 \mathrm{mmol}), \mathrm{Na}_{2} \mathrm{CO}_{3}(79 \mathrm{mg}, 0.74 \mathrm{mmol})$ and CSI $(43 \mu \mathrm{L}, 0.49 \mathrm{mmol})$ in $\mathrm{CHCl}_{3}(2 \mathrm{~mL})$ at $0{ }^{\circ} \mathrm{C}$. The reaction mixture was purified by column chromatography (Hexane/EtOAc $=3: 1)$ to give $53 \mathrm{mg}(89 \%)$ inseparable mixture of $(1 R, 2 S)$-methyl $N$-(2-methoxy-1-p-methoxyphenylbut-3-enyl)carbamate (2) and (1S,2S)methyl $N$-(2-methoxy-1-p-methoxyphenylbut-3-enyl)carbamate (4) (7.6:1 ratio) as a white solid.

The above general procedure was followed using $(1 R, 2 S)$-1,2-dimethoxy-1-p-methoxyphenylbut-3-ene (3) (50 mg, $0.23 \mathrm{mmol}), \mathrm{Na}_{2} \mathrm{CO}_{3}(79 \mathrm{mg}, 0.74 \mathrm{mmol})$ and $\mathrm{CSI}(43 \mu \mathrm{L}, 0.49 \mathrm{mmol})$ in $\mathrm{Et}_{2} \mathrm{O}(2 \mathrm{~mL})$ at $0{ }^{\circ} \mathrm{C}$. The reaction mixture was purified by column chromatography (Hexane/EtOAc $=3: 1$ ) to give $57 \mathrm{mg}(96 \%)$ inseparable mixture of $(1 R, 2 S)$-methyl $N$-(2-methoxy-1-p-methoxyphenylbut-3-enyl)carbamate (2) and $(1 S, 2 S)$ methyl $N$-(2-methoxy-1-p-methoxyphenylbut-3-enyl)carbamate (4) (12:1 ratio) as a white solid.

The above general procedure was followed using (1R,2S)-1,2-dimethoxy-1-p-methoxyphenylbut-3-ene (3) (50 mg, $0.23 \mathrm{mmol}), \mathrm{Na}_{2} \mathrm{CO}_{3}(79 \mathrm{mg}, 0.74 \mathrm{mmol})$ and CSI $(43 \mu \mathrm{L}, 0.49 \mathrm{mmol})$ in toluene $(2 \mathrm{~mL})$ at $0^{\circ} \mathrm{C}$. The reaction mixture was purified by column chromatography (Hexane/EtOAc $=3: 1$ ) to give $56 \mathrm{mg}(94 \%)$ inseparable mixture of $(1 R, 2 S)$-methyl $N$-(2-methoxy-1-p-methoxyphenylbut-3-enyl)carbamate (2) and (1S,2S)methyl $N$-(2-methoxy-1-p-methoxyphenylbut-3-enyl)carbamate (4) (16:1 ratio) as a white solid.

The above general procedure was followed using (1R,2S)-1,2-dimethoxy-1-p-methoxyphenylbut-3-ene (3) (50 mg, $0.23 \mathrm{mmol}), \mathrm{Na}_{2} \mathrm{CO}_{3}(79 \mathrm{mg}, 0.74 \mathrm{mmol})$ and CSI $(43 \mu \mathrm{L}, 0.49 \mathrm{mmol})$ in $\mathrm{CCl}_{4}(2 \mathrm{~mL})$ at $0{ }^{\circ} \mathrm{C}$. The reaction mixture was purified by column chromatography (Hexane/EtOAc $=3: 1)$ to give $54 \mathrm{mg}(91 \%)$ inseparable mixture of $(1 R, 2 S)$-methyl $N$-(2-methoxy-1-p-methoxyphenylbut-3-enyl)carbamate (2) and (1S,2S)methyl $N$-(2-methoxy-1-p-methoxyphenylbut-3-enyl)carbamate (4) (18:1 ratio) as a white solid.

The above general procedure was followed using $(1 R, 2 S)$-1,2-dimethoxy-1-p-methoxyphenylbut-3-ene (3) (50 mg, $0.23 \mathrm{mmol}), \mathrm{Na}_{2} \mathrm{CO}_{3}(79 \mathrm{mg}, 0.74 \mathrm{mmol})$ and CSI $(43 \mu \mathrm{L}, 0.49 \mathrm{mmol})$ in hexane $(2 \mathrm{~mL})$ at $0{ }^{\circ} \mathrm{C}$. The reaction mixture was purified by column chromatography (Hexane/EtOAc $=3: 1$ ) to give $40 \mathrm{mg}(67 \%$ ) inseparable mixture of $(1 R, 2 S)$-methyl $N$-(2-methoxy-1-p-methoxyphenylbut-3-enyl)carbamate (2) and (1S,2S)- 
methyl $N$-(2-methoxy-1-p-methoxyphenylbut-3-enyl)carbamate (4) (13:1 ratio) as a white solid.

The above general procedure was followed using $(1 R, 2 S)$-1,2-dimethoxy-1-p-methoxyphenylbut-3-ene (3) (50 mg, $0.23 \mathrm{mmol}), \mathrm{Na}_{2} \mathrm{CO}_{3}(79 \mathrm{mg}, 0.74 \mathrm{mmol})$ and CSI $(43 \mu \mathrm{L}, 0.49 \mathrm{mmol})$ in hexane $(2 \mathrm{~mL})$ at $-78{ }^{\circ} \mathrm{C}$. The reaction mixture was purified by column chromatography (Hexane/EtOAc $=3: 1)$ to give $54 \mathrm{mg}(91 \%)$ inseparable mixture of $(1 R, 2 S)$-methyl $N$-(2-methoxy-1-p-methoxyphenylbut-3-enyl)carbamate (2) and (1S,2S)methyl $N$-(2-methoxy-1-p-methoxyphenylbut-3-enyl)carbamate (4) (15:1 ratio) as a white solid.

\section{$(1 R, 2 R)$-Methyl $N$-(1-p-methoxyphenyl-2-methylbut-3-enyl)carbamate (6) and $(1 S, 2 R)$-methyl $N$-(1-p- methoxyphenyl-2-methylbut-3-enyl)carbamate (7).}

The above general procedure was followed using (1R,2R)-1-methoxy-1-p-methoxyphenyl-2-methylbut-3ene (5) (50 mg, $0.24 \mathrm{mmol}), \mathrm{Na}_{2} \mathrm{CO}_{3}(58 \mathrm{mg}, 0.55 \mathrm{mmol})$ and $\mathrm{CSI}(32 \mu \mathrm{L}, 0.36 \mathrm{mmol})$ in $\mathrm{CH}_{2} \mathrm{Cl}_{2}(2 \mathrm{~mL})$ at -78 ${ }^{\circ} \mathrm{C}$. The reaction mixture was purified by column chromatography (Hexane/EtOAc $\left.=3: 1\right)$ to give $55 \mathrm{mg}(91 \%)$ inseparable mixture of $(1 R, 2 R)$-methyl $N$-(1-p-methoxyphenyl-2-methylbut-3-enyl)carbamate (6) and $(1 S, 2 R)$ methyl $N$-(1-p-methoxyphenyl-2-methylbut-3-enyl)carbamate (7) $(1: 1.8$ ratio) as a white solid.

(1R,2R)-Methyl N-(1-p-methoxyphenyl-2-methylbut-3-enyl)carbamate (6).

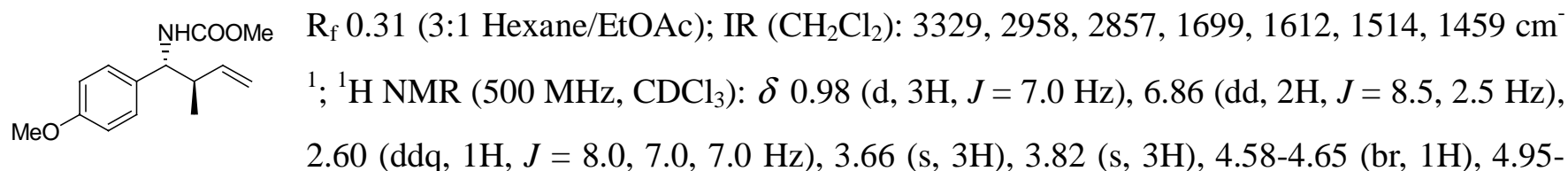

5.05 (br, 1H), 5.06 (dd, 1H, $J=10.5,1.5 \mathrm{~Hz}), 5.09$ (dd, 1H, $J=16.0,1.5 \mathrm{~Hz}), 5.60$ (ddd, 1H, $J=16.0,10.5$, $8.0 \mathrm{~Hz}), 6.86(\mathrm{dd}, 2 \mathrm{H}, J=8.5,2.5 \mathrm{~Hz}), 7.12(\mathrm{dd}, 2 \mathrm{H}, J=8.5,2.5 \mathrm{~Hz}) ;{ }^{13} \mathrm{C} \mathrm{NMR}\left(125 \mathrm{MHz}, \mathrm{CDCl}_{3}\right): \delta 17.41$, $43.42,52.38,55.50,58.72,113.85,116.35,128.53,132.68,139.96,156.54,159.01$. HRMS (CI) calcd for $\mathrm{C}_{14} \mathrm{H}_{19} \mathrm{NO}_{3}+\mathrm{H}(\mathrm{M}+\mathrm{H})^{+} 250.1443$. Found: 250.1451 .

(1S,2R)-Methyl N-(1-p-methoxyphenyl-2-methylbut-3-enyl)carbamate (7).

$\mathrm{Hz}), 3.64(\mathrm{~s}, 3 \mathrm{H}), 3.81(\mathrm{~s}, 3 \mathrm{H}), 4.42-4.53(\mathrm{br}, 1 \mathrm{H}), 4.95-5.05(\mathrm{br}, 1 \mathrm{H}), 5.08(\mathrm{dd}, 1 \mathrm{H}, J=$
$10.5,1.5 \mathrm{~Hz}), 5.11(\mathrm{dd}, 1 \mathrm{H}, J=16.5,1.5 \mathrm{~Hz}), 5.74(\mathrm{ddd}, 1 \mathrm{H}, J=16.5,10.5,8.0 \mathrm{~Hz}), 6.87$ $(\mathrm{dd}, 2 \mathrm{H}, J=8.5,2.5 \mathrm{~Hz}), 7.17(\mathrm{dd}, 2 \mathrm{H}, J=8.5,2.5 \mathrm{~Hz}) ;{ }^{13} \mathrm{C} \mathrm{NMR}\left(125 \mathrm{MHz}, \mathrm{CDCl}_{3}\right): \delta 16.79,43.97$, $52.36,55.51,59.01,114.24,116.47,128.02,133.76,139.98,156.64,158.98$. 
The above general procedure was followed using (1R,2R)-1-methoxy-1-p-methoxyphenyl-2-methylbut-3ene (5) (50 mg, $0.24 \mathrm{mmol}), \mathrm{Na}_{2} \mathrm{CO}_{3}(58 \mathrm{mg}, 0.55 \mathrm{mmol})$ and CSI (32 $\left.\mu \mathrm{L}, 0.36 \mathrm{mmol}\right)$ in toluene $(2 \mathrm{~mL})$ at -78 ${ }^{\circ} \mathrm{C}$. The reaction mixture was purified by column chromatography (Hexane/EtOAc $\left.=3: 1\right)$ to give $50 \mathrm{mg}(83 \%)$ inseparable mixture of $(1 R, 2 R)$-methyl $N$-(1-p-methoxyphenyl-2-methylbut-3-enyl)carbamate (6) and $(1 S, 2 R)$ methyl $N$-(1-p-methoxyphenyl-2-methylbut-3-enyl)carbamate (7) $(1: 1.7$ ratio) as a white solid.

The above general procedure was followed using (1R,2R)-1-methoxy-1-p-methoxyphenyl-2-methylbut-3ene (5) (50 mg, $0.24 \mathrm{mmol}), \mathrm{Na}_{2} \mathrm{CO}_{3}(58 \mathrm{mg}, 0.55 \mathrm{mmol})$ and CSI (32 $\left.\mu \mathrm{L}, 0.36 \mathrm{mmol}\right)$ in hexane $(2 \mathrm{~mL})$ at -78 ${ }^{\circ} \mathrm{C}$. The reaction mixture was purified by column chromatography (Hexane/EtOAc $\left.=3: 1\right)$ to give $49 \mathrm{mg}(81 \%)$ inseparable mixture of $(1 R, 2 R)$-methyl $N$-(1-p-methoxyphenyl-2-methylbut-3-enyl)carbamate (6) and $(1 S, 2 R)$ methyl $N$-(1-p-methoxyphenyl-2-methylbut-3-enyl)carbamate (7) $(1: 1.6$ ratio) as a white solid.

\section{Total Synthesis of (-)-Cytoxazone}

\section{(2R,3R)-2-Methoxy-3-methoxycarbonylamino-3-p-methoxyphenylpropan-1-ol (10).}

Ozone was bubbled through a solution of $(1 R, 2 S)$-methyl $N$-(2-methoxy-1-p-methoxyphenylbut-3enyl)carbamate (2) $(0.70 \mathrm{~g}, 2.64 \mathrm{mmol})$ in anhydrous $\mathrm{CH}_{2} \mathrm{Cl}_{2}(10 \mathrm{~mL})$ and $\mathrm{MeOH}(20 \mathrm{~mL})$ at $-78{ }^{\circ} \mathrm{C}$ until blue color persisted, the excess was then purged out with $\mathrm{N}_{2}$ until decolorization, and $\mathrm{NaBH}_{4}(1.00 \mathrm{~g}, 26.38 \mathrm{mmol})$ was added at $-78{ }^{\circ} \mathrm{C}$. The reaction mixture was slowly warmed to $0{ }^{\circ} \mathrm{C}$, stirred at $0{ }^{\circ} \mathrm{C}$ for $1 \mathrm{~h}$ and concentrated in vacuo. The residue was dissolved in $\mathrm{H}_{2} \mathrm{O}(30 \mathrm{~mL})$ and EtOAc $(50 \mathrm{~mL})$, the organic layer was separated and washed with $\mathrm{H}_{2} \mathrm{O}$ and brine, dried over $\mathrm{MgSO}_{4}$ and concentrated in vacuo. The residue was purified by column chromatography $($ Hexane/ EtOAc $=1: 2)$ to give $0.67 \mathrm{~g}(94 \%)$ of $(2 R, 3 R)$-2-methoxy-3-methoxycarbonylamino3-p-methoxyphenyl propan-1-ol (10) as a colorless syrup.

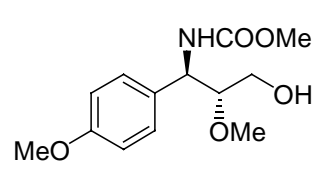

$\mathrm{R}_{\mathrm{f}} 0.21\left(1: 2\right.$ Hexane/EtOAc); $[\alpha]_{\mathrm{D}}{ }^{25}-52.1^{\mathrm{o}}\left(c\right.$ 0.2, $\left.\mathrm{CDCl}_{3}\right) ; \mathrm{IR}\left(\mathrm{CH}_{2} \mathrm{Cl}_{2}\right): 3390,2951$, 2836, 1704, 1612, 1514, $1463 \mathrm{~cm}^{-1} ;{ }^{1} \mathrm{H}$ NMR (500 MHz, $\mathrm{CDCl}_{3}$ ): $\delta$ 2.20-2.28 (br, 1H), 3.43 (ddd, 1H, $J=7.5,5.5,3.5 \mathrm{~Hz}$ ), 3.46 (s, 3H), 3.50-3.56 (br d, 1H, $J=2.0 \mathrm{~Hz}$ ), 3.68 (s, 3H), 3.64-3.75 (br, 1H), 3.82 (s, 3H), 4.97-5.03 (br, 1H), 5.82 (br d, 1H, J = 8.5 Hz), 6.89 (dd, 2H, J = 7.0, 2.0 $\mathrm{Hz}), 7.25(\mathrm{dd}, 2 \mathrm{H}, J=7.0,2.0 \mathrm{~Hz}) ;{ }^{13} \mathrm{C} \mathrm{NMR}\left(125 \mathrm{MHz}, \mathrm{CDCl}_{3}\right): \delta 52.59,55.24,55.55,58.21,61.19,82.84$, 114.28, 128.42, 131.52, 157.15, 159.24; HRMS (CI) calcd for $\mathrm{C}_{13} \mathrm{H}_{19} \mathrm{NO}_{5}+\mathrm{H}(\mathrm{M}+\mathrm{H})^{+}$270.1341. Found: 270.1341 . 


\section{(2R,3R)-3-Methoxycarbonylamino-3-p-methoxyphenylpropane-1,2-diol (11).}

To a solution of (2R,3R)-2-methoxy-3-methoxycarbonylamino-3-p-methoxyphenylpropan-1-ol (10) (0.50 g, $1.86 \mathrm{mmol})$ in anhydrous $\mathrm{CH}_{2} \mathrm{Cl}_{2}(20 \mathrm{~mL})$ was added $\mathrm{BBr}_{3}\left(2.04 \mathrm{~mL}, 2.04 \mathrm{mmol}, 1.0 \mathrm{M}\right.$ in $\left.\mathrm{CH}_{2} \mathrm{Cl}_{2}\right)$ at $0{ }^{\circ} \mathrm{C}$ under $\mathrm{N}_{2}$. The reaction mixture was stirred at $0{ }^{\circ} \mathrm{C}$ for $30 \mathrm{~min}$, quenched with saturated $\mathrm{NaHCO}_{3}$ aqueous solution. The organic layer was separated and the aqueous layer was extracted with $\mathrm{CH}_{2} \mathrm{Cl}_{2}(10 \mathrm{~mL} \times 3)$. The combined organic layer was washed with $\mathrm{H}_{2} \mathrm{O}$ and brine, dried over $\mathrm{MgSO}_{4}$ and concentrated in vacuo. The residue was purified by column chromatography (Hexane/EtOAc $=1: 4)$ to give $0.38 \mathrm{~g}(80 \%)$ of $(2 R, 3 R)-3$ methoxycarbonylamino-3-p-methoxyphenylpropane-1,2-diol (11) as a white solid.

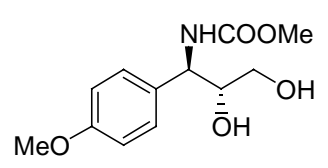

$\mathrm{R}_{\mathrm{f}} 0.31\left(6: 1 \mathrm{CHCl}_{3} / \mathrm{MeOH}\right) ; \operatorname{mp} 81 \sim 84{ }^{\circ} \mathrm{C} ;[\alpha]_{\mathrm{D}}{ }^{25}-60.0^{\circ}\left(c 0.1, \mathrm{CDCl}_{3}\right) ; \mathrm{IR}\left(\mathrm{CH}_{2} \mathrm{Cl}_{2}\right)$ : 3347, 2953, 2836, 1699, 1612, 1514, $1462 \mathrm{~cm}^{-1} ;{ }^{1} \mathrm{H}$ NMR (500 MHz, $\left.\mathrm{CDCl}_{3}\right): \delta$ 2.422.48 (br, 1H), 2.62-2.72 (br, 1H), 3.67 (s, 3H), 3.60-3.75 (br, 2H), 3.80 (s, 3H), 3.84-3.93

(br d, 1H, $J=13.5 \mathrm{~Hz}), 4.72(\mathrm{dd}, 1 \mathrm{H}, J=8.0,7.0 \mathrm{~Hz}$ ), 5.40-5.46 (br, 1H), 6.90 (dd, 2H, $J=9.5,2.0 \mathrm{~Hz}), 7.25$ $(\mathrm{dd}, 2 \mathrm{H}, J=9.5,2.0 \mathrm{~Hz}) ;{ }^{13} \mathrm{C} \mathrm{NMR}\left(125 \mathrm{MHz}, \mathrm{CDCl}_{3}\right): \delta 52.73,55.54,57.04,63.53,74.04,114.48,128.75$, 130.94, 157.48, 159.53; Anal. Calcd for $\mathrm{C}_{12} \mathrm{H}_{17} \mathrm{NO}_{4}$ : C, 56.46; H, 6.71; N, 5.49. Found: C, 56.34; H, 6.73; N, 5.39 .

\section{(4R,5R)-5-Hydroxymethyl-4-p-methoxyphenyl-1,3-oxazolidin-2-one (1).}

To a solution of (2R,3R)-3-methoxycarbonylamino-3-p-methoxyphenylpropane-1,2-diol (11) (0.30 g, 1.18 $\mathrm{mmol})$ in anhydrous THF (12 mL) was added $\mathrm{NaH}\left(52 \mathrm{mg}, 1.29 \mathrm{mmol}, 60 \%\right.$ in mineral oil) at $0{ }^{\circ} \mathrm{C}$ under $\mathrm{N}_{2}$. The reaction mixture was stirred at $0{ }^{\circ} \mathrm{C}$ for $2 \mathrm{~h}$ and quenched with $\mathrm{H}_{2} \mathrm{O}(10 \mathrm{~mL})$, then extracted with EtOAc $(10 \mathrm{~mL} \times 2)$. The organic layer was washed with $\mathrm{H}_{2} \mathrm{O}$ and brine, dried over $\mathrm{MgSO}_{4}$ and concentrated in vacuo. The residue was purified by column chromatography (Hexane/EtOAc $=1: 4)$ to give $0.25 \mathrm{~g}(95 \%)$ of $(4 R, 5 R)-5$ hydroxymethyl-4-p-methoxyphenyl-1,3-oxazolidin-2-one (1) as a white solid.

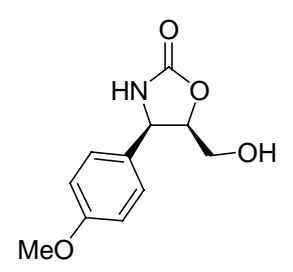

$\mathrm{R}_{\mathrm{f}} 0.37\left(6: 1 \mathrm{CHCl}_{3} / \mathrm{MeOH}\right) ; \mathrm{mp} 118 \sim 120{ }^{\circ} \mathrm{C} ;[\alpha]_{\mathrm{D}}{ }^{24}-70.9^{\circ}$ (c $\left.0.1, \mathrm{MeOH}\right)$, lit. ${ }^{1} \mathrm{mp}$ $118 \sim 120^{\circ} \mathrm{C} ;[\alpha]_{\mathrm{D}}{ }^{25}-71^{\circ}(\mathrm{c} 0.1, \mathrm{MeOH}) ;{ }^{1} \mathrm{H}$ NMR (500 MHz, DMSO-d 6 ): $\delta$ 2.94-2.99 (m, 2H), 3.75(s, 3H), $4.70(\mathrm{ddd}, 1 \mathrm{H}, J=8.5,7.5,4.5 \mathrm{~Hz}), 4.81(\mathrm{t}, 1 \mathrm{H}, J=5.0 \mathrm{~Hz}), 4.90(\mathrm{~d}, 1 \mathrm{H}, J$ $=8.5 \mathrm{~Hz}), 6.93(\mathrm{dd}, 2 \mathrm{H}, J=8.5,3.0 \mathrm{~Hz}), 7.15(\mathrm{dd}, 2 \mathrm{H}, J=8.5,3.0 \mathrm{~Hz}), 8.03-8.06(\mathrm{br}, 1 \mathrm{H})$;

${ }^{13} \mathrm{C}$ NMR (125 MHz, DMSO-d $\left.{ }_{6}\right): \delta$ 55.81, 56.92, 61.76, 80.77, 114.39, 128.74, 129.99, 159.47, 159.72; HRMS (CI) calcd for $\mathrm{C}_{11} \mathrm{H}_{13} \mathrm{NO}_{4}+\mathrm{H}(\mathrm{M}+\mathrm{H})^{+} 2224.0923$. Found: 224.0925. 


$$
\text { (1) }
$$

$896 \cdot \tau$

so $\varepsilon^{\prime} z$

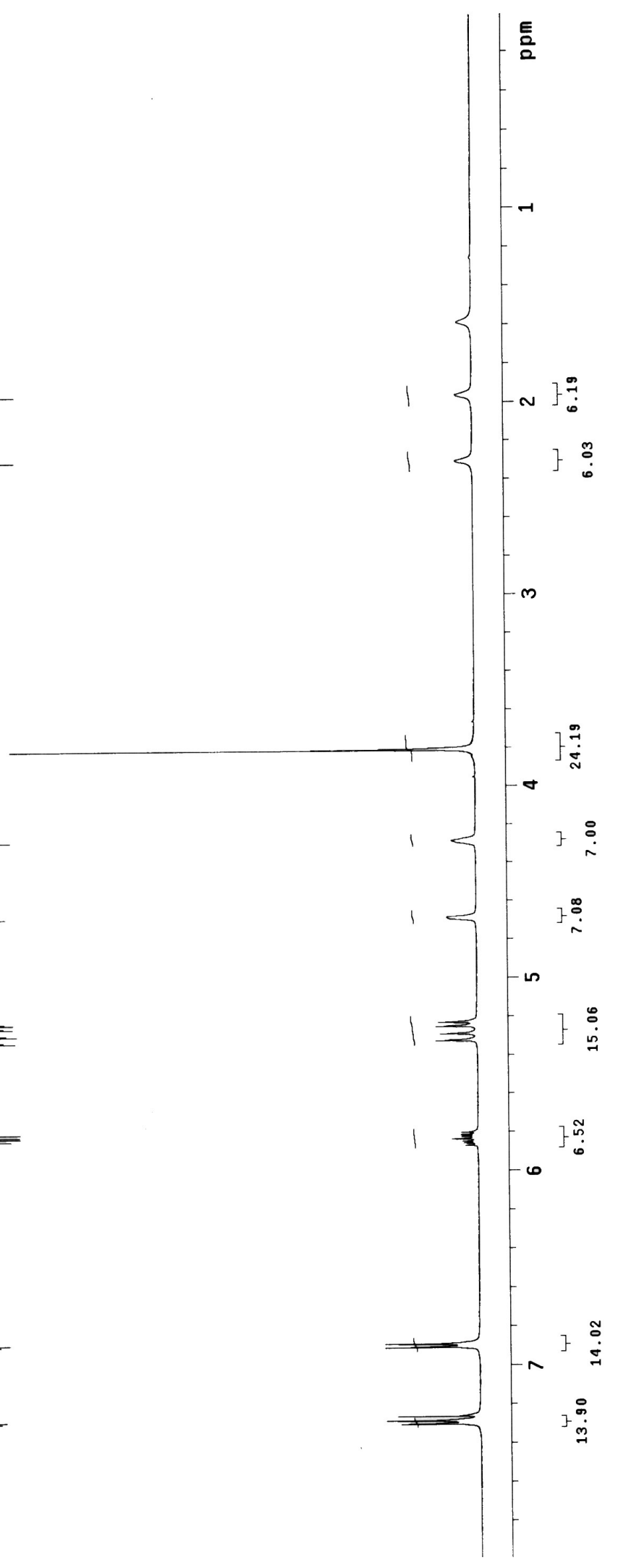



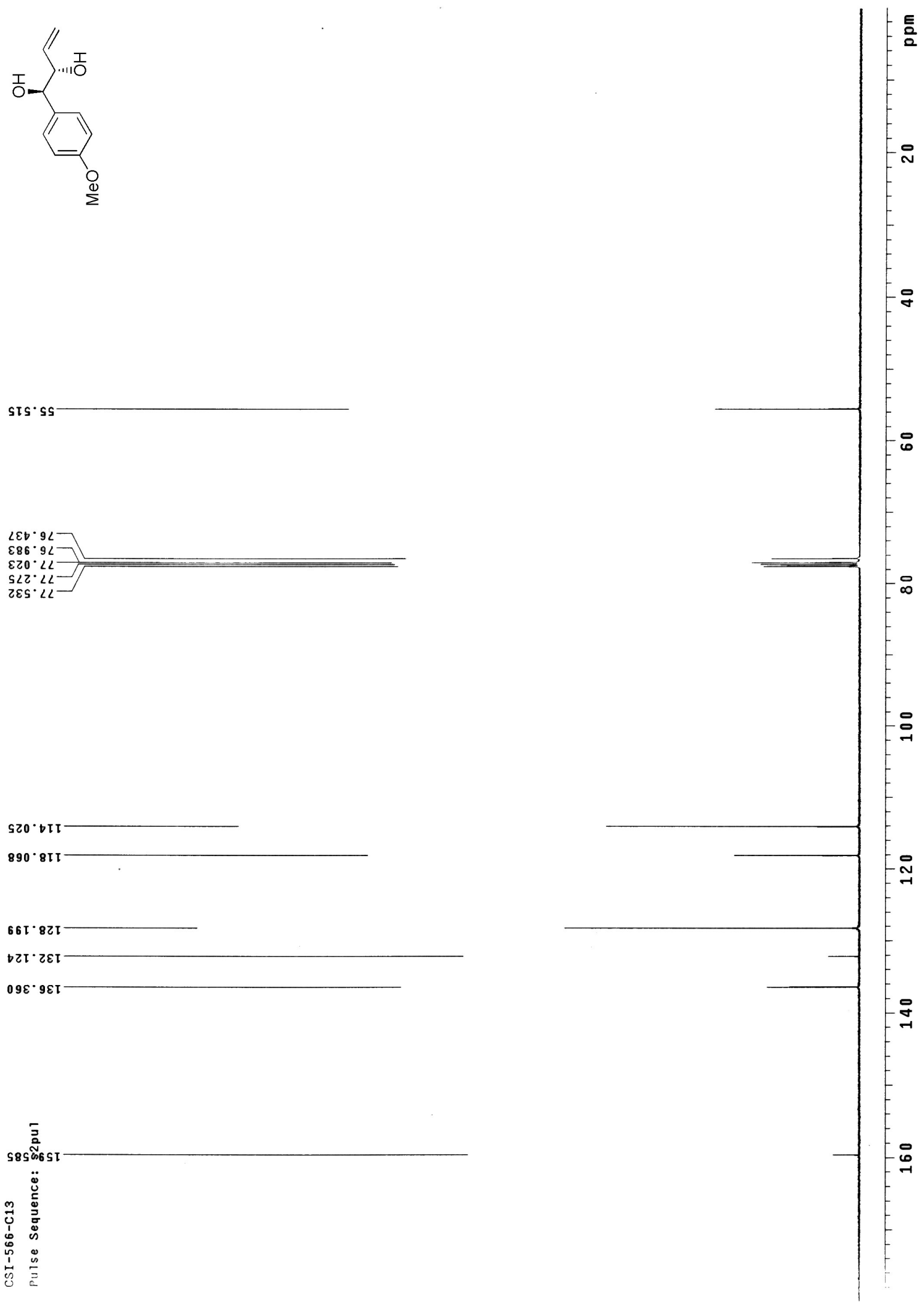


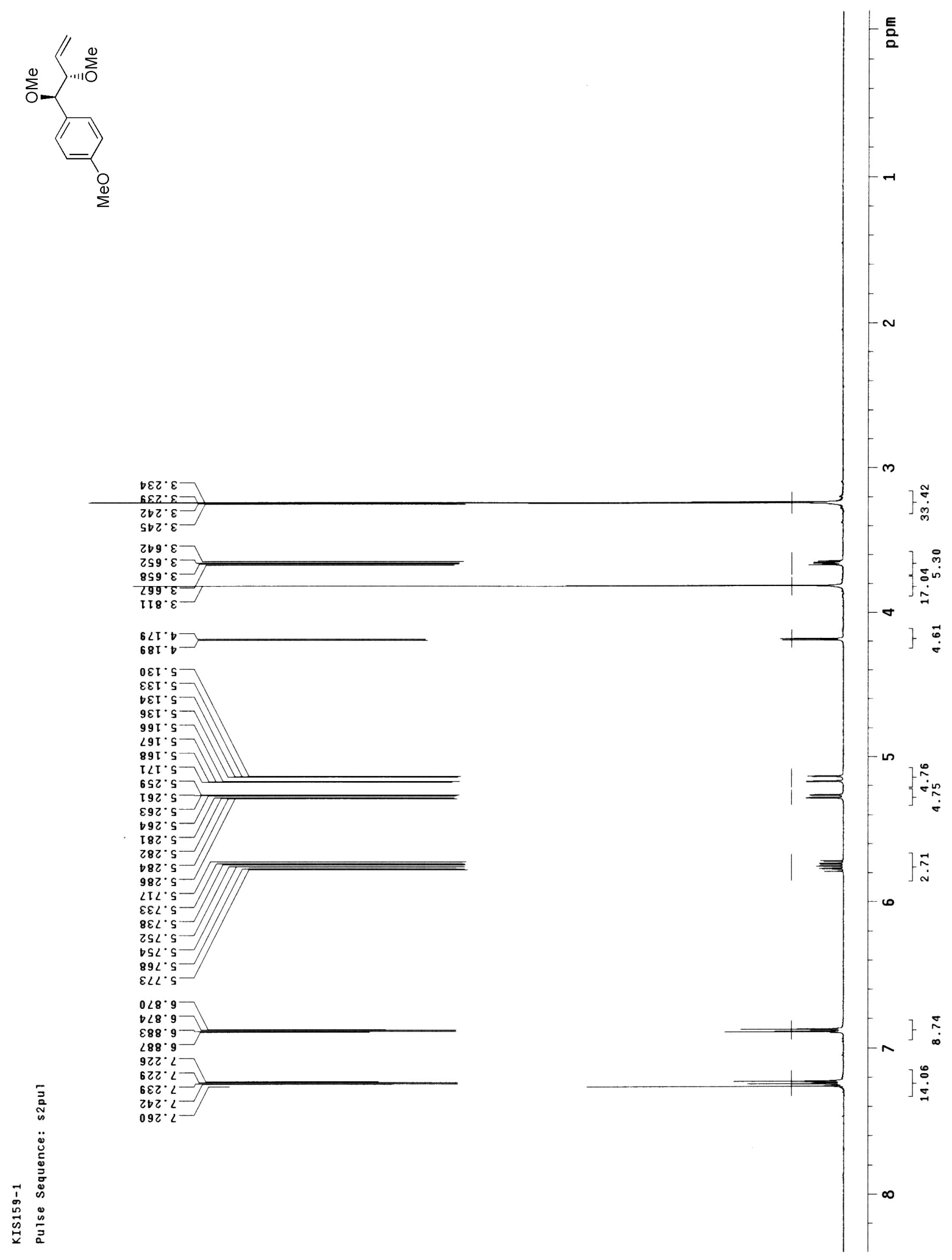




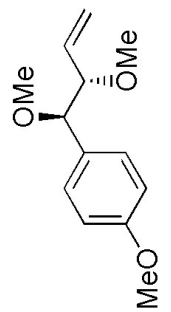

$261 \cdot 5 \mathrm{~S}$

$808^{\circ} .95$

DS6.9S

$\angle 0<\cdot 9 L$

$000 \cdot L 2$

$60 S^{\circ} \mathrm{s}$
$\varepsilon \varepsilon \varepsilon^{\circ} \cdot 98$

$\varepsilon I t \cdot \varepsilon I T$

S9I. 6 II

$686^{\circ} 82 \mathrm{I}$

$285^{\circ} 0 \varepsilon \mathrm{I}$

$069^{\circ} \sqcup \varepsilon$ เ

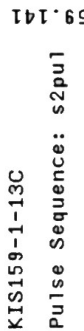

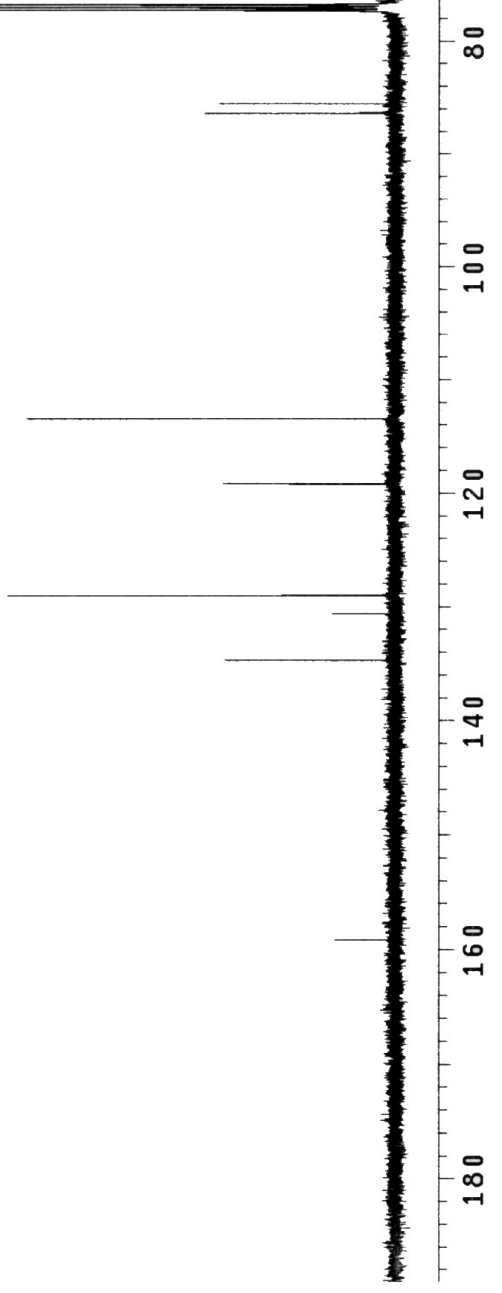




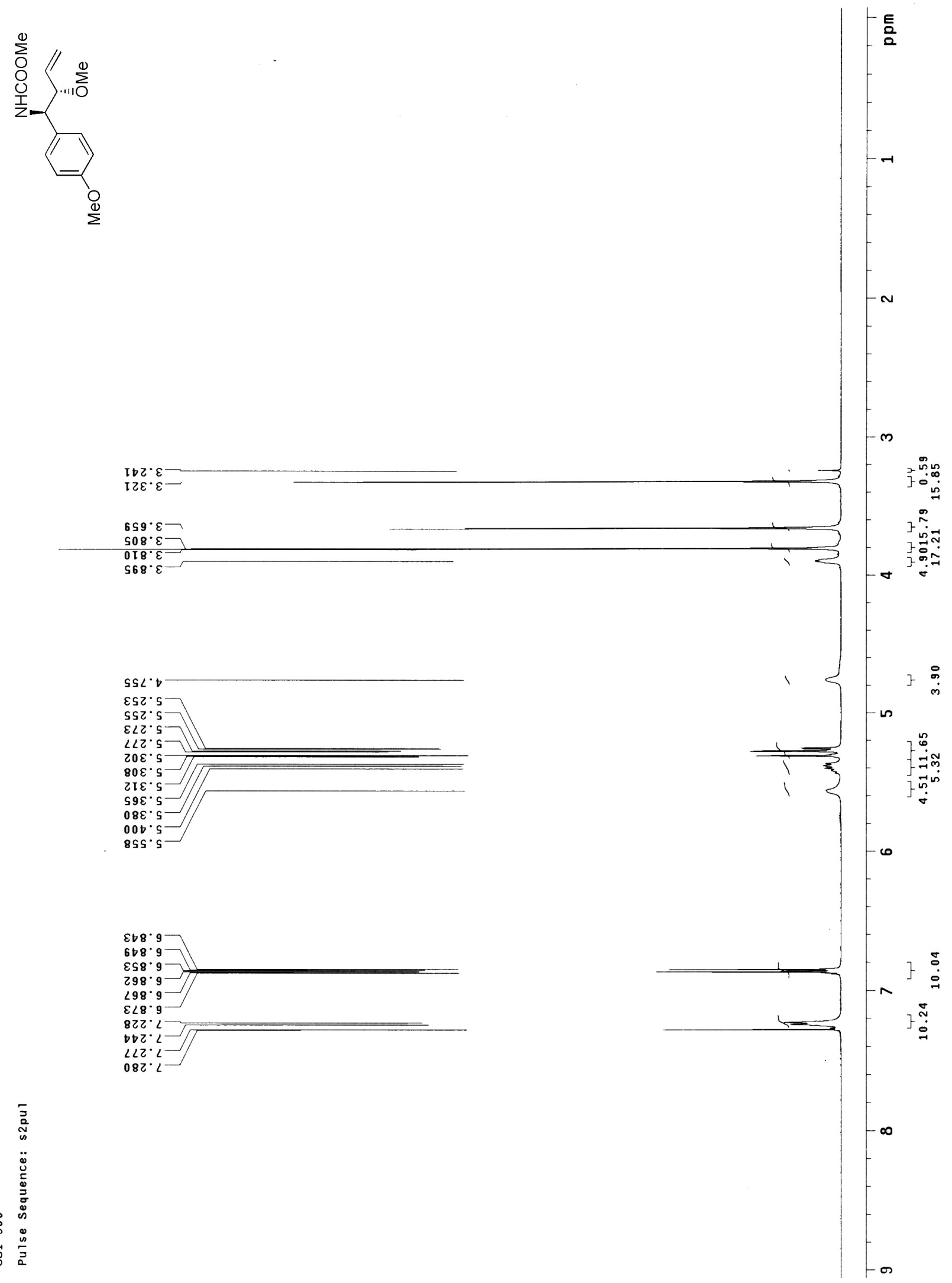



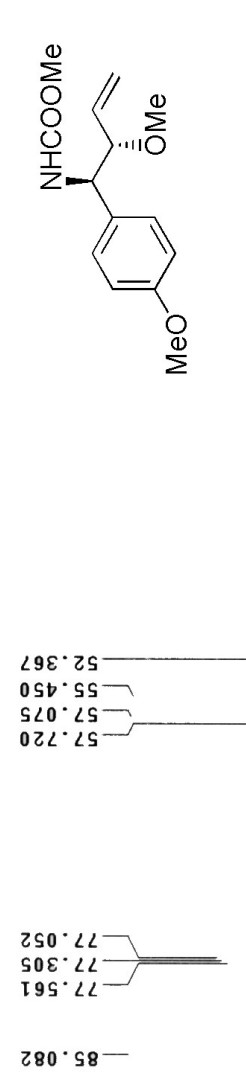

$\varepsilon L L \cdot \varepsilon I I-$

$2 I L \cdot 6 I I-$

I $0 E^{\circ} 62 I$

S9I. IEI

$090 \cdot \varsigma \varepsilon \mathrm{I}$

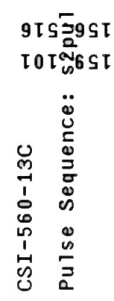




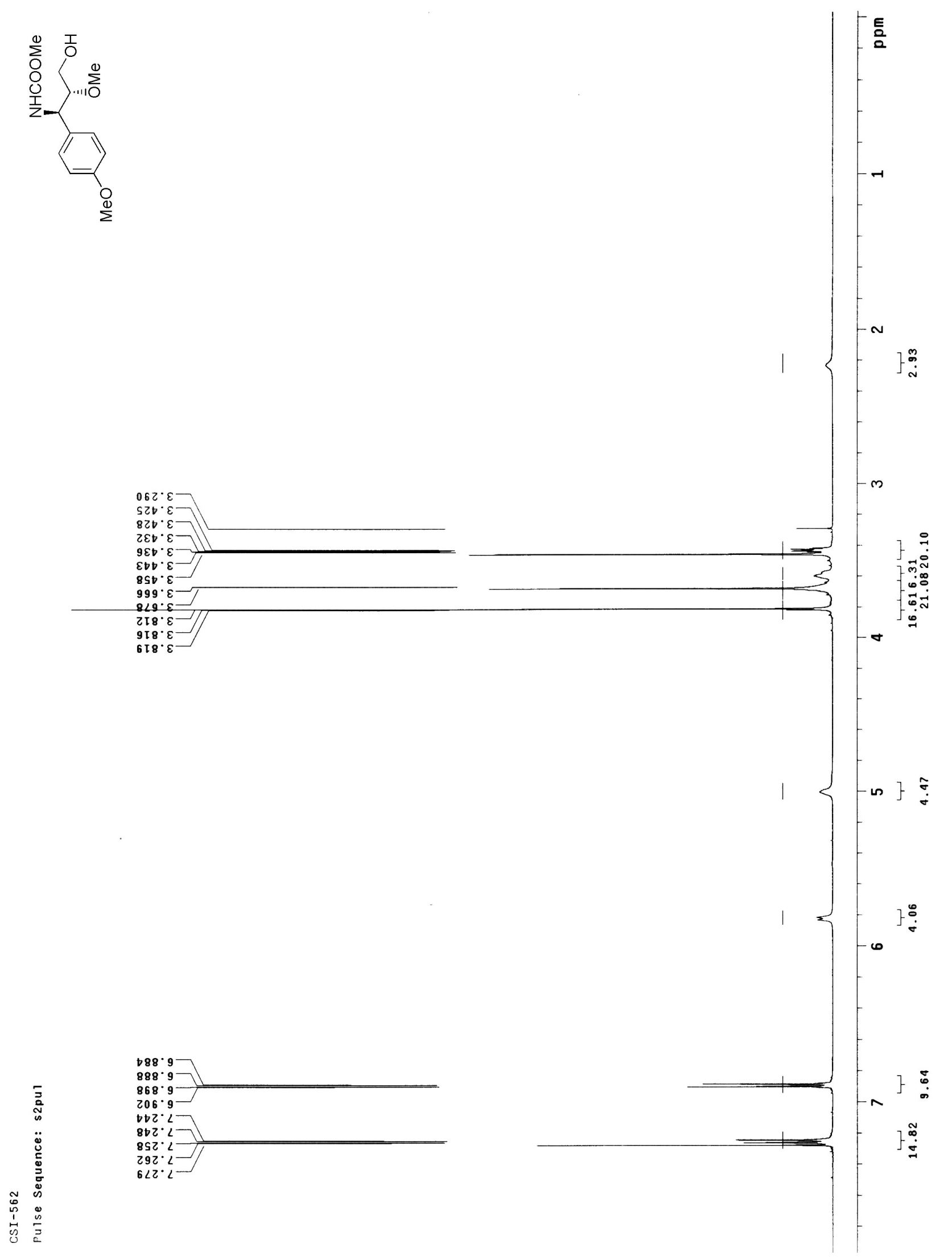



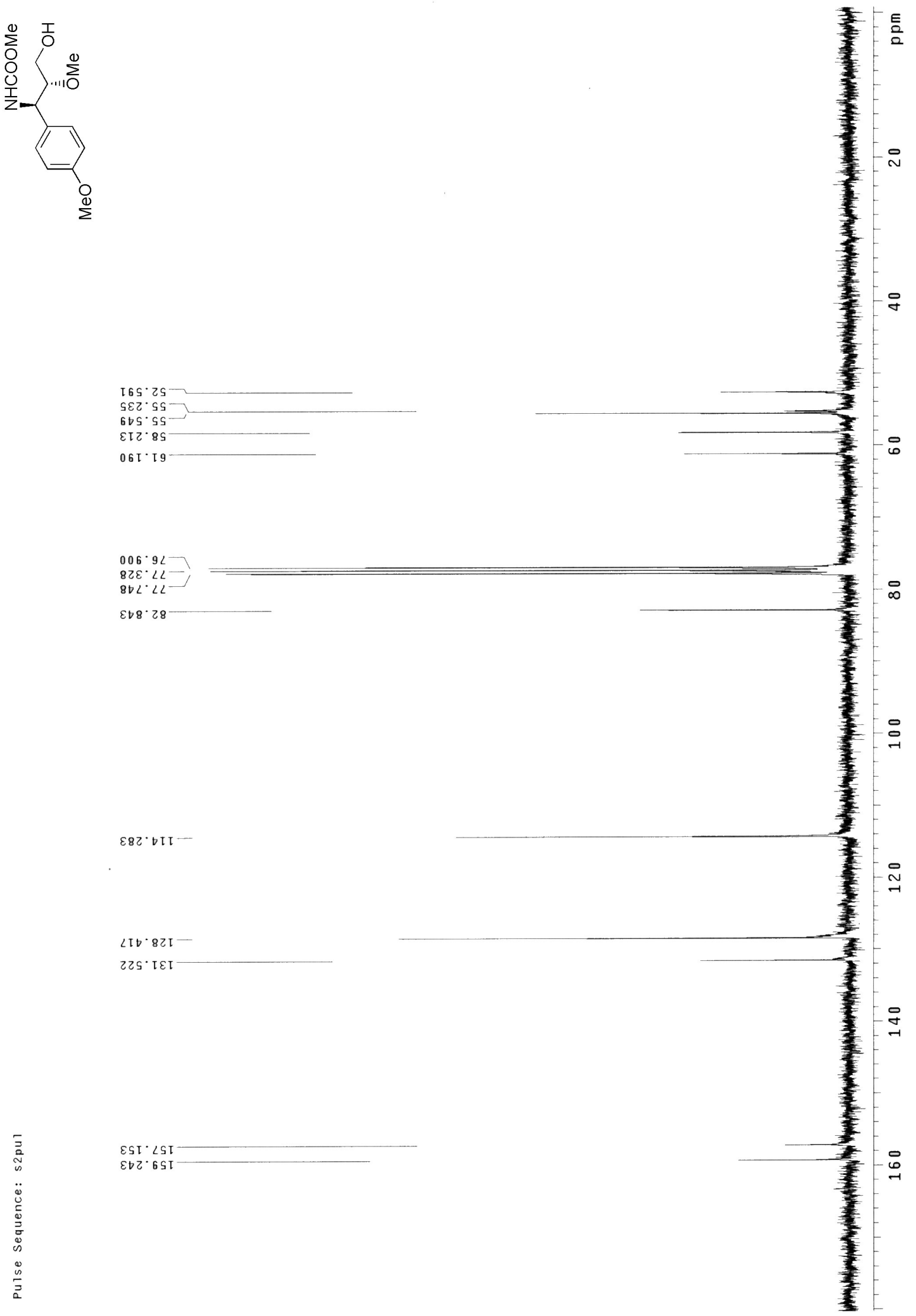

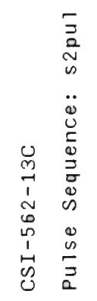

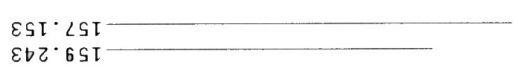

16 


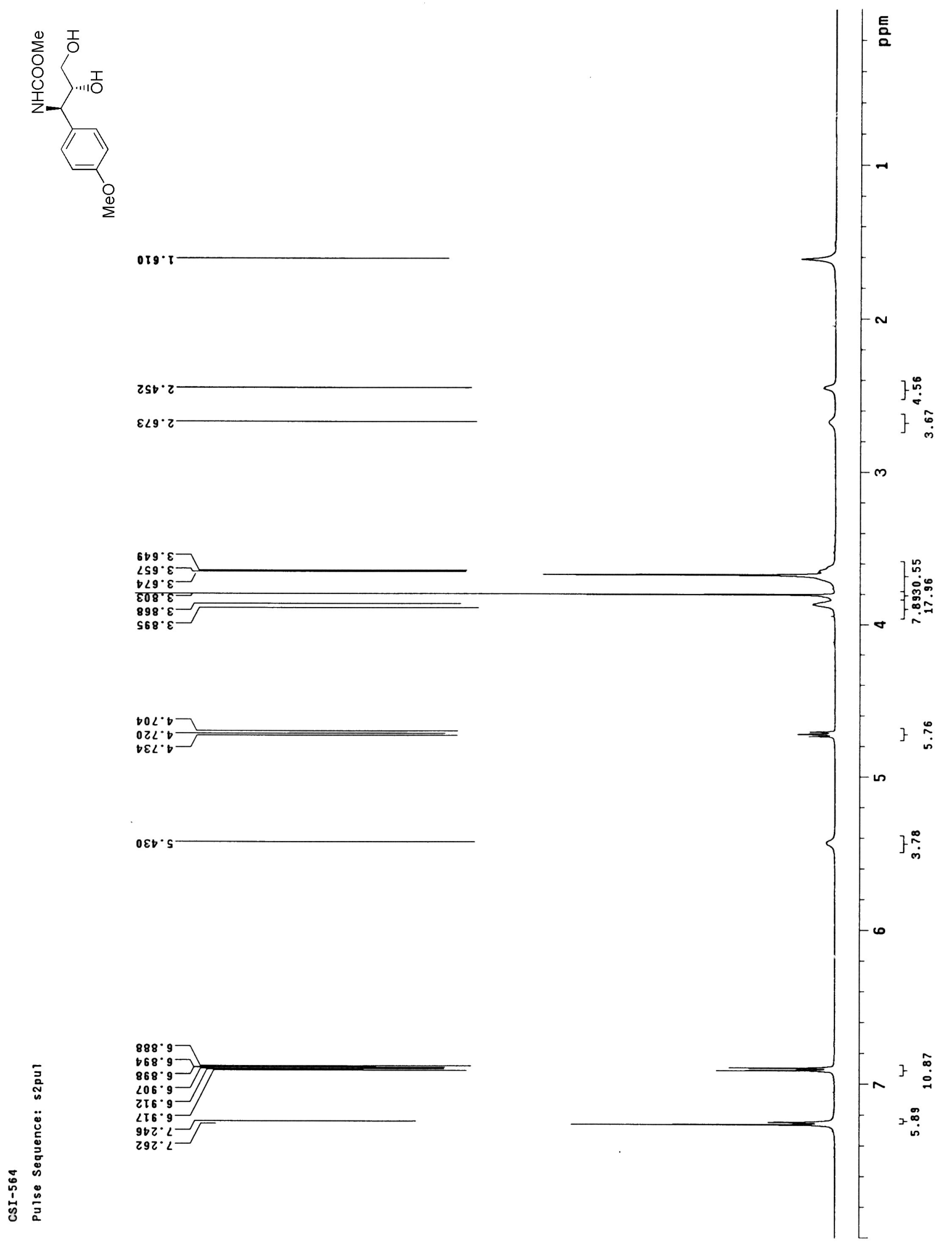



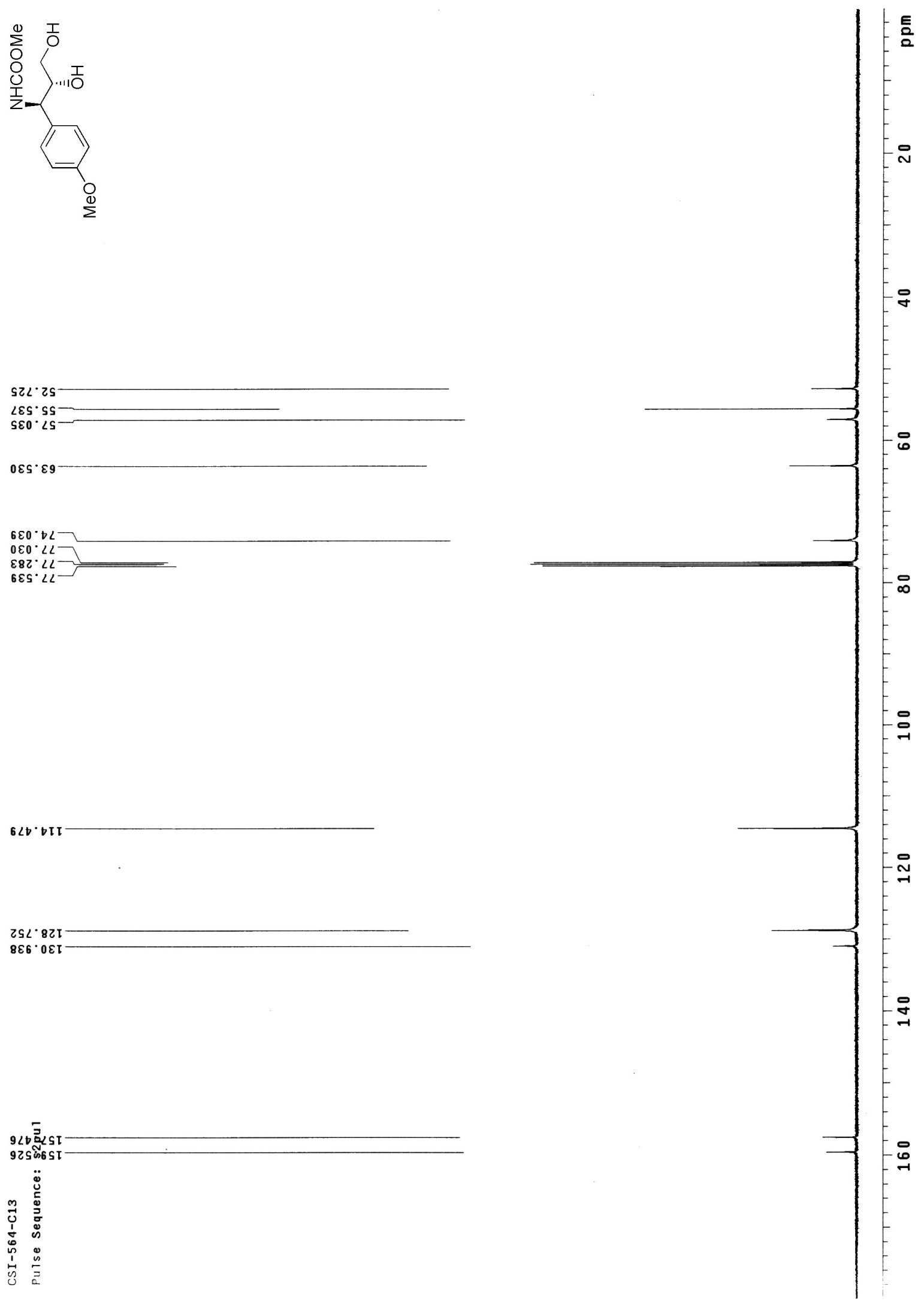

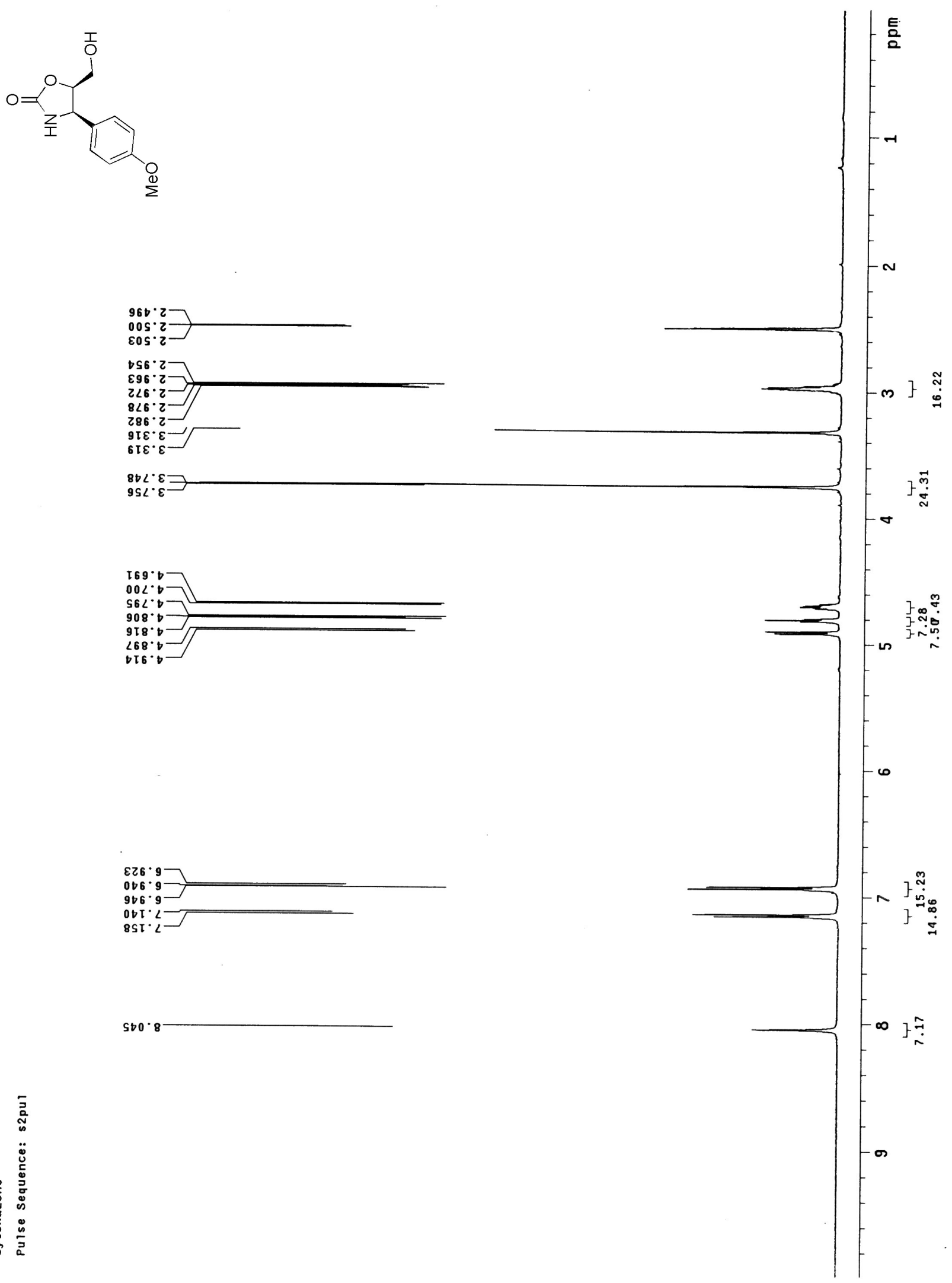

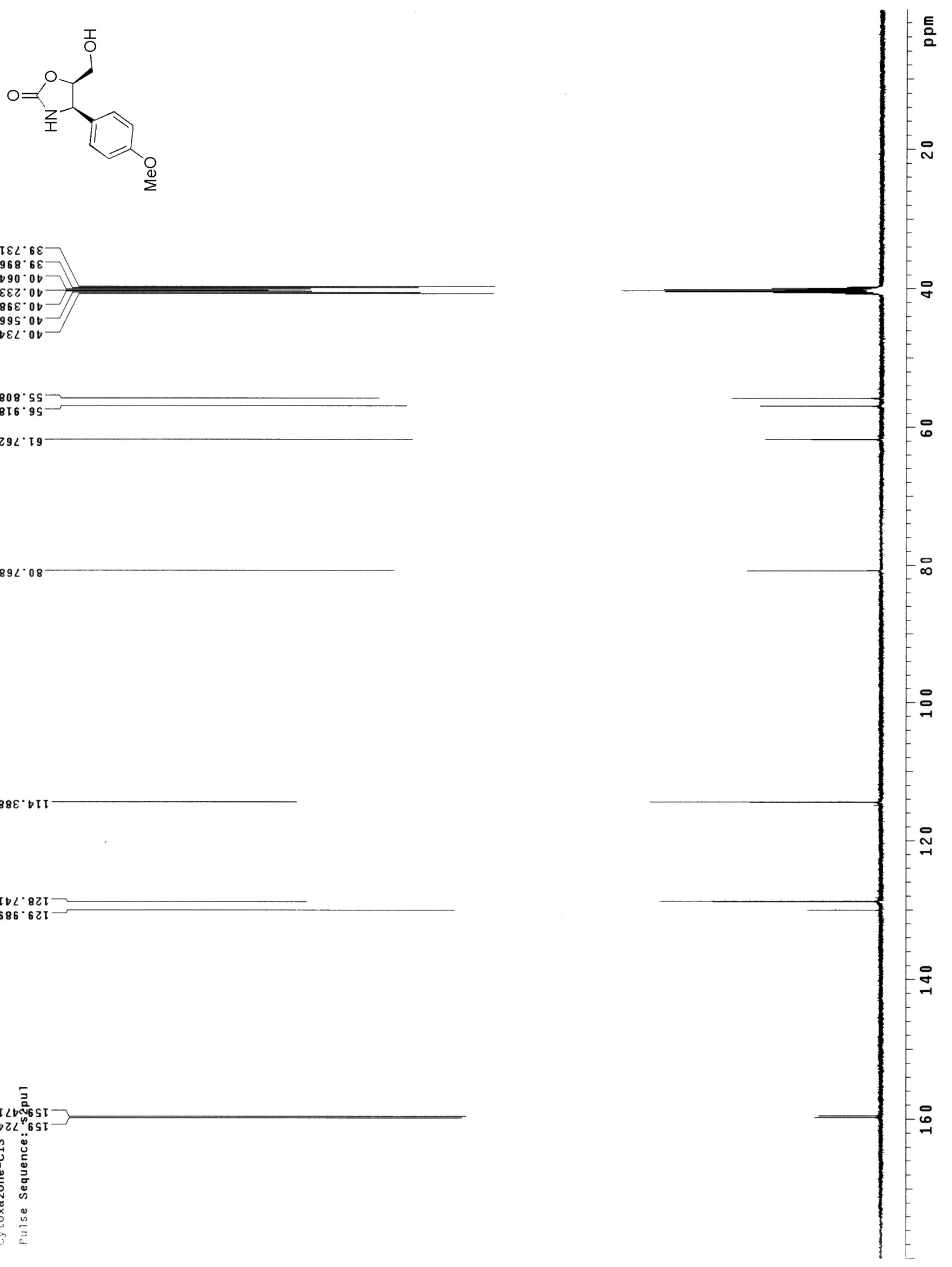

I $\varepsilon L \cdot 6 \varepsilon$

$968.6 \varepsilon$

$b 90^{\circ} 0 b$

$86 \varepsilon^{\circ} \circ b$

995. $0 t$

$\checkmark E L \cdot O t$

$808^{\circ}$ SS

816.95

$29 L^{\circ} 19$

$89 L \cdot 08$

$88 \varepsilon^{\circ} \downarrow I I$

It $L \circ 82$

686.621

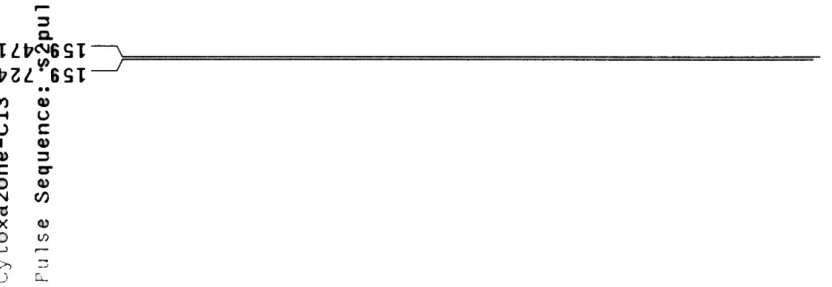

